\title{
Virtual Screening of DPP-4 Inhibitors Using QSAR- Based Artificial Intelligence and Molecular Docking of Hit Compounds to DPP-8 and DPP-9 Enzymes
}

\section{CURRENT STATUS: UNDER REVIEW}

BMC Bioinformatics $\triangle$ BMC series

\section{Oky Hermansyah}

Universitas Indonesia

ORCiD: https://orcid.org/0000-0002-7701-3809

Alhadi Bustamam

Universitas Indonesia

Arry Yanuar

ح arry.yanuar@ui.ac.idCorresponding Author

ORCiD: https://orcid.org/0000-0001-8895-9010

DOI:

$10.21203 / \mathrm{rs} .2 .22282 / \mathrm{v} 1$

\section{SUBJECT AREAS}

Bioinformatics

\section{KEYWORDS}

Artificial Intelligence, DPP-4, KNIME, Machine Learning, QSAR, Virtual Screening 
Abstract

Background: Dipeptidyl Peptidase-4 (DPP-4) inhibitors are becoming an essential drug in the treatment of type 2 diabetes mellitus, but some classes of these drugs have side effects such as joint pain that can become severe to pancreatitis. It is thought that these side effects appear related to their inhibition against enzymes DPP-8 and DPP-9.

Objective: This study aims to find DPP-4 inhibitor hit compounds that are selective against the DPP-8 and DPP-9 enzymes. By building a virtual screening workflow using the Quantitative Structure-Activity Relationship (QSAR) method based on artificial intelligence (AI), millions of molecules from the database can be screened for the DPP-4 enzyme target with a faster time compared to other screening methods.

Result: Five regression machine learning algorithms and four classification machine learning algorithms were used to build virtual screening workflows. The algorithm that qualifies for the regression QSAR model was Support Vector regression with R 2 pred 0.78, while the classification QSAR model was Random Forest with $92.21 \%$ accuracy. The virtual screening results of more than 10 million molecules from the database, obtained 2,716 hit compounds with pIC50 above 7.5. Molecular docking results of several potential hit compounds to the DPP-4, DPP-8 and DPP-9 enzymes, obtained $\mathrm{CH} 0002$ hit compound that has a high inhibitory potential against the DPP-4 enzyme and low inhibition of the DPP-8 and DPP-9 enzymes.

Conclusion: This research was able to produce DPP-4 inhibitor hit compounds that are potential to DPP-4 and selective to DPP-8 and DPP-9 enzymes so that they can be further developed in the DPP-4 inhibitors discovery. The resulting virtual screening workflow can be applied to the discovery of hit compounds on other targets. Keywords: Artificial Intelligence; DPP-4; KNIME; Machine Learning; QSAR;

\section{Virtual Screening}

\section{Full-text}

Due to technical limitations, full-text HTML conversion of this manuscript could not be completed. However, the manuscript can be downloaded and accessed as a PDF. Tables

Table 1. Internal validation results on the classification model 


\begin{tabular}{lccccccccc}
\hline \multicolumn{1}{c}{ Models } & TP & FP & TN & FN & Sensitivity & Specificity & F-Measure & Precision & Acc \\
\hline Deep Learning & 891 & 93 & 786 & 75 & 0.9224 & 0.8942 & 0.9138 & 0.9055 & 0.5 \\
Random Forest & 925 & 105 & 774 & 41 & 0.9576 & 0.8805 & 0.9269 & 0.8981 & 0.5 \\
SVM & 952 & 457 & 422 & 14 & 0.9855 & 0.4801 & 0.8017 & 0.6757 & 0. \\
XGBoost & 907 & 93 & 786 & 59 & 0.9389 & 0.8942 & 0.9227 & 0.9070 & 0.5 \\
\hline
\end{tabular}

*TP $=$ True Positive; FP = False Positive; TN = True Negative; FN = False Negative

Table 2. External validation statistical parameters of various models

\begin{tabular}{|c|c|c|c|c|c|c|}
\hline Metric & DL & XGBoost & MLR & RF & SVR & Standard \\
\hline$R_{(c v)}^{2}$ & 0.6920 & 0.7530 & 0.5939 & 0.7532 & 0.7607 & $>0.5^{a}$ \\
\hline$R^{2}{ }_{(\text {pred })}$ & 0.5910 & 0.7617 & 0.6013 & 0.7668 & 0.7761 & $>0.6^{a}$ \\
\hline MSE (cv) & 0.7686 & 0.6163 & 1.0134 & 0.6157 & 0.5971 & - \\
\hline \multirow[t]{3}{*}{ MSE (pred) } & 1.0370 & 0.6043 & 1.0109 & 0.5914 & 0.5679 & - \\
\hline & 0.2564 & 0.6914 & 0.4597 & 0.6319 & 0.7281 & - \\
\hline & 0.5911 & 0.7618 & 0.6014 & 0.7668 & 0.7762 & - \\
\hline () / & 0.5672 & 0.0924 & 0.2422 & 0.1847 & 0.0624 & $<0.1^{a}$ \\
\hline \multirow[t]{2}{*}{ () / } & 0.0023 & 0.0000 & 0.0086 & 0.0107 & 0.0006 & $<0.1^{a}$ \\
\hline & 0.3347 & 0.0704 & 0.1417 & 0.1349 & 0.0481 & $<0.3^{a}$ \\
\hline k & 0.9979 & 1.0024 & 0.9976 & 1.0005 & 0.9975 & $0.85 \leq k \leq 1.15^{a}$ \\
\hline \multirow[t]{5}{*}{$k^{\prime}$} & 0.9797 & 0.9845 & 0.9805 & 0.9867 & 0.9902 & $0.85 \leq k^{\prime} \leq 1.15^{a}$ \\
\hline & 0.2760 & 0.5181 & 0.3665 & 0.4272 & 0.5668 & - \\
\hline & 0.5686 & 0.7618 & 0.5586 & 0.6874 & 0.7563 & - \\
\hline & 0.4223 & 0.6400 & 0.4625 & 0.5573 & 0.6616 & $>0.5^{b}$ \\
\hline & 0.2925 & 0.2437 & 0.1920 & 0.2602 & 0.1895 & $<0.2^{b}$ \\
\hline Model Predictive & Fail & Fail & Fail & Fail & Yes & \\
\hline
\end{tabular}

\footnotetext{
$* a$ = standard Golbraikh \& Tropsha (2002)

$\mathrm{b}=$ standard Roy, Kar \& Das (2015)
}

Table 3. External validation results on the classification model 


\begin{tabular}{|c|c|c|c|c|c|c|c|c|c|c|}
\hline Models & TP & FP & TN & $\mathbf{F N}$ & Sensitivity & Specificity & \multicolumn{2}{|c|}{ F-measure } & \multicolumn{2}{|l|}{ Precision } \\
\hline Deep Learning & 215 & 25 & 212 & 10 & 0.9556 & 0.8945 & $\begin{array}{c}0.92 \\
47\end{array}$ & 0.8958 & & .9242 \\
\hline Random Forest & 219 & 29 & 208 & 6 & 0.9733 & 0.8776 & $\begin{array}{c}0.92 \\
60\end{array}$ & 0.8831 & & .9242 \\
\hline SVM & 221 & 137 & 100 & 4 & 0.9822 & 0.4219 & $\begin{array}{c}0.75 \\
81\end{array}$ & 0.6173 & & .6948 \\
\hline XGBoost & 215 & 26 & 211 & 10 & 0.9556 & 0.8903 & $\begin{array}{c}0.92 \\
27\end{array}$ & 0.8921 & & .9221 \\
\hline
\end{tabular}

Table 4. Performance of QSAR method workflows that are automated on various targets

\begin{tabular}{|c|c|c|c|c|c|c|c|c|}
\hline Target & Models & $R^{2}(c v)$ & MSE & $\mathbf{R}^{2}$ (Pred) & Dataset & Curation & Training & Validation \\
\hline \multirow{5}{*}{$\begin{array}{l}\text { Beta-1 } \\
\text { adrenergic } \\
\text { receptor } \\
\text { (CHEMBL213) }\end{array}$} & $\begin{array}{l}\text { Deep } \\
\text { Learning }\end{array}$ & 0.6601 & 0.4265 & 0.9134 & \multirow[t]{5}{*}{1508} & \multirow[t]{5}{*}{620} & \multirow[t]{5}{*}{446} & \multirow[t]{5}{*}{496} \\
\hline & MLR & 0.1570 & 1.0578 & -0.2724 & & & & \\
\hline & $\begin{array}{l}\text { Random } \\
\text { Forest }\end{array}$ & 0.7349 & 0.3326 & 0.6462 & & & & \\
\hline & SVR & 0.7312 & 0.3373 & 0.6515 & & & & \\
\hline & XGBoost & 0.7099 & 0.3641 & 0.6676 & & & & \\
\hline \multirow{5}{*}{$\begin{array}{l}\text { Sigma Opioid } \\
\text { receptor } \\
\text { (CHEMBL233) }\end{array}$} & $\begin{array}{l}\text { Deep } \\
\text { Learning }\end{array}$ & 0.6730 & 0.6113 & 0.0736 & \multirow[t]{5}{*}{2280} & \multirow[t]{5}{*}{1157} & \multirow[t]{5}{*}{832} & \multirow[t]{5}{*}{925} \\
\hline & MLR & 0.4672 & 0.9959 & 0.5693 & & & & \\
\hline & $\begin{array}{l}\text { Random } \\
\text { Forest }\end{array}$ & 0.7725 & 0.4253 & 0.7318 & & & & \\
\hline & SVR & 0.7543 & 0.4593 & 0.7311 & & & & \\
\hline & XGBoost & 0.7453 & 0.4762 & 0.7422 & & & & \\
\hline
\end{tabular}

Table 5. Molecular docking results of hit compounds to DPP-4, DPP-8, and DPP-9 enzymes.

\begin{tabular}{|c|c|c|c|c|c|c|c|}
\hline \multicolumn{2}{|c|}{ No. } & \multirow{2}{*}{$\begin{array}{c}\text { Macromolecule } \\
\text { (PDB ID) } \\
5 K B Y\end{array}$} & \multirow{2}{*}{$\begin{array}{l}\text { Ligand } \\
6 \text { RL1510 }\end{array}$} & \multirow{2}{*}{$\begin{array}{c}\begin{array}{c}\text { Binding } \\
\text { Energy }\end{array} \\
-9.66\end{array}$} & \multirow{2}{*}{$\begin{array}{c}\boldsymbol{K i} \\
82.49 \\
\end{array}$} & \multirow{2}{*}{$\begin{array}{c}\text { Unit } \\
\mathrm{nM}\end{array}$} & \multirow{2}{*}{$\begin{array}{l}\text { Molecule } \\
\text { Trelagliptin }\end{array}$} \\
\hline $\bar{A}$ & 1 & & & & & & \\
\hline & 2 & & $\mathrm{CH} 0001$ & -9.42 & 125.21 & $\mathrm{nM}$ & \\
\hline & 3 & & $\mathrm{CH} 0002$ & -9.67 & 81.13 & $\mathrm{nM}$ & \\
\hline & 4 & & $\mathrm{CH} 0003$ & -11.27 & 5.51 & $\mathrm{nM}$ & \\
\hline & 5 & & MP0001 & -5.67 & 69420 & $\mathrm{nM}$ & \\
\hline & 6 & & MP0002 & -8.54 & 551.45 & $\mathrm{nM}$ & \\
\hline & 7 & & MP0005 & -4.55 & 459580 & $\mathrm{nM}$ & \\
\hline
\end{tabular}




\begin{tabular}{|c|c|c|c|c|c|c|c|}
\hline & \multicolumn{2}{|l|}{8} & PC0001 & -6.68 & 12780 & \multicolumn{2}{|l|}{$\mathrm{nM}$} \\
\hline & 9 & & PC0002 & -7.13 & 5910 & $\mathrm{nM}$ & \\
\hline & 10 & & PC0003 & -8.43 & 658 & $\mathrm{nM}$ & \\
\hline \multirow[t]{10}{*}{$B$} & 1 & $2 \mathrm{ONC}$ & SY1800 & -10.43 & 22.83 & $\mathrm{nM}$ & Alogliptin \\
\hline & 2 & & $\mathrm{CH} 0001$ & -9.51 & 106.54 & $\mathrm{nM}$ & \\
\hline & 3 & & $\mathrm{CH} 0002$ & -9.78 & 67.62 & $\mathrm{nM}$ & \\
\hline & 4 & & $\mathrm{CH} 0003$ & -11.46 & 4 & $\mathrm{nM}$ & \\
\hline & 5 & & MP0001 & -5.27 & 136760 & $\mathrm{nM}$ & \\
\hline & 6 & & MP0002 & -8.5 & 586.04 & $\mathrm{nM}$ & \\
\hline & 7 & & MP0005 & -4.7 & 358230 & $\mathrm{nM}$ & \\
\hline & 8 & & PC0001 & -6.43 & 19500 & $\mathrm{nM}$ & \\
\hline & 9 & & PC0002 & -6.77 & 10940 & $\mathrm{nM}$ & \\
\hline & 10 & & PC0003 & -7.97 & 1440 & $\mathrm{nM}$ & \\
\hline \multirow[t]{10}{*}{$\mathrm{C}$} & 1 & 4PNZ & $2 \mathrm{VH} 802$ & -10.37 & 25.12 & $n M$ & Omargliptin \\
\hline & 2 & & $\mathrm{CH} 0001$ & -9.78 & 67.96 & $\mathrm{nM}$ & \\
\hline & 3 & & $\mathrm{CH} 0002$ & -8.22 & 940.40 & $\mathrm{nM}$ & \\
\hline & 4 & & $\mathrm{CH} 0003$ & -8.91 & 293.62 & $\mathrm{nM}$ & \\
\hline & 5 & & MP0001 & -4.89 & 261080 & $\mathrm{nM}$ & \\
\hline & 6 & & MP0002 & -7 & 7420 & $\mathrm{nM}$ & \\
\hline & 7 & & MP0005 & -3.69 & 1980000 & $\mathrm{nM}$ & \\
\hline & 8 & & PC0001 & -6.86 & 9350 & $\mathrm{nM}$ & \\
\hline & 9 & & PC0002 & -6.65 & 13280 & $\mathrm{nM}$ & \\
\hline & 10 & & PC0003 & -7.66 & 2410 & $\mathrm{nM}$ & \\
\hline \multirow[t]{10}{*}{$\bar{D}$} & 1 & $3 \mathrm{KWF}$ & B1Q1 & -9.75 & 71.41 & $\mathrm{nM}$ & Carmegliptin \\
\hline & 2 & & $\mathrm{CH} 0001$ & -9.05 & 234.16 & $\mathrm{nM}$ & \\
\hline & 3 & & $\mathrm{CH} 0002$ & -9.21 & 177.65 & $\mathrm{nM}$ & \\
\hline & 4 & & $\mathrm{CH} 0003$ & -10.11 & 39.1 & $\mathrm{nM}$ & \\
\hline & 5 & & MP0001 & -4.04 & 1090000 & $\mathrm{nM}$ & \\
\hline & 6 & & MP0002 & -7.41 & 3730 & $\mathrm{nM}$ & \\
\hline & 7 & & MP0005 & -4.16 & 897610 & $\mathrm{nM}$ & \\
\hline & 8 & & PC0001 & -6.94 & 8170 & $\mathrm{nM}$ & \\
\hline & 9 & & PC0002 & -6.35 & 22310 & $\mathrm{nM}$ & \\
\hline & 10 & & PC0003 & -7.59 & 2720 & $\mathrm{nM}$ & \\
\hline \multirow[t]{4}{*}{$\bar{E}$} & 1 & $6 \mathrm{HP} 8$ & GK2901 & -6.69 & 12490 & $\mathrm{nM}$ & DPP-8 \\
\hline & 2 & & $\mathrm{CHOOO1}$ & -8.88 & 310.16 & $\mathrm{nM}$ & \\
\hline & 3 & & $\mathrm{CH} 0002$ & -8.08 & 1190 & $\mathrm{nM}$ & \\
\hline & 4 & & $\mathrm{CH} 0003$ & -9.93 & 52.98 & $\mathrm{nM}$ & \\
\hline
\end{tabular}




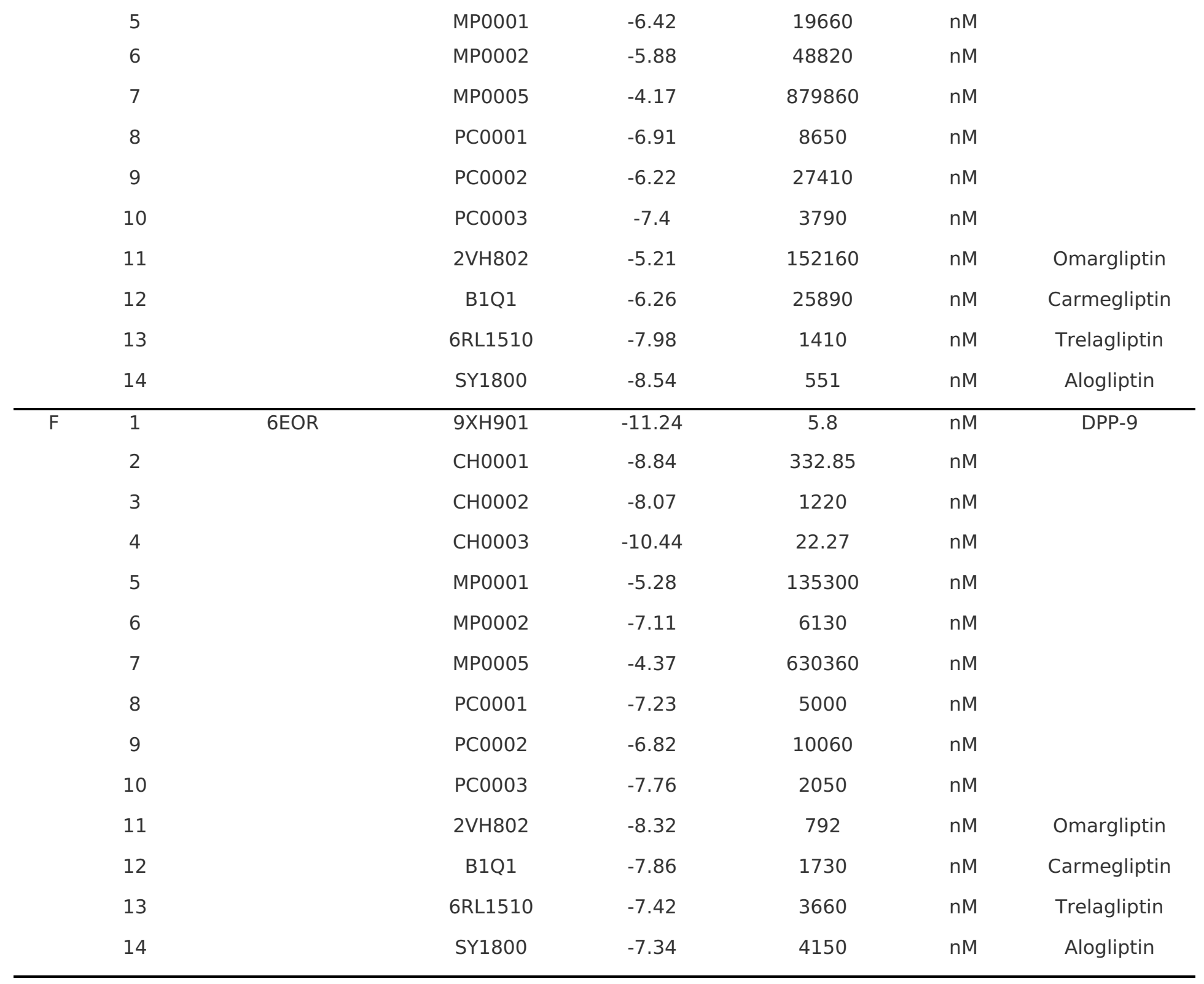

Figures

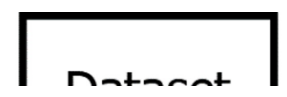




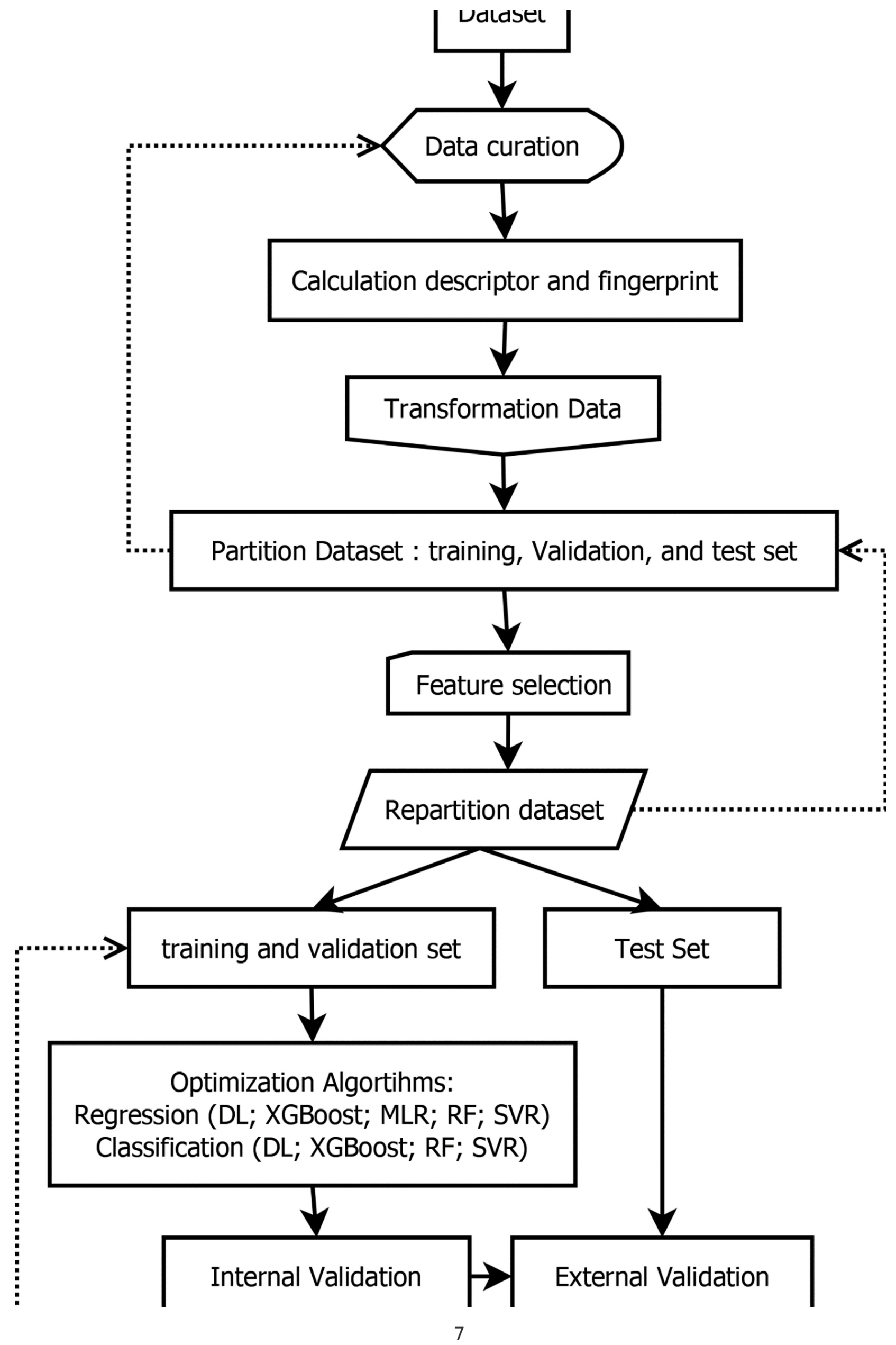




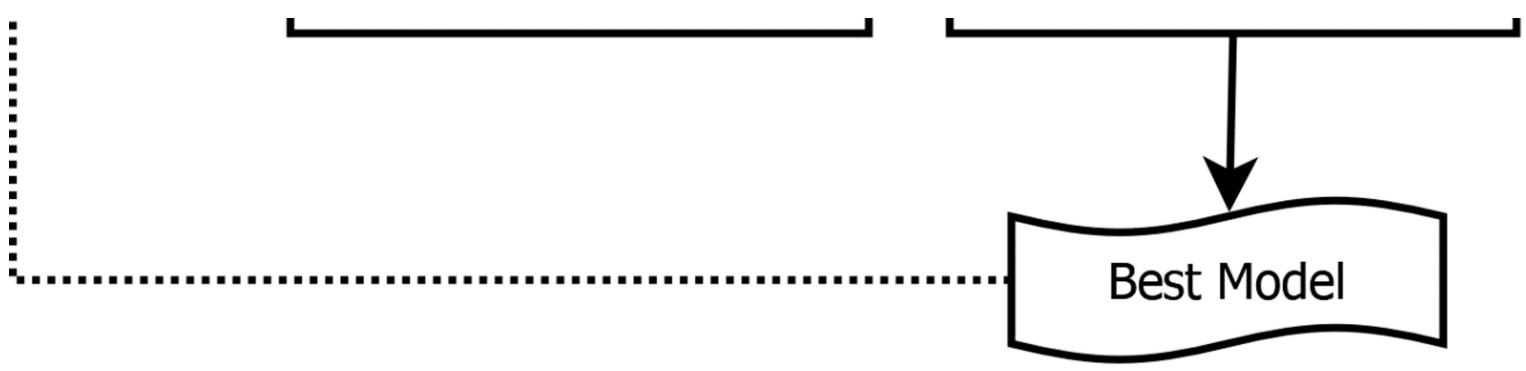

Figure 1

the workflow of QSAR modeling DPP 4 inhibitors

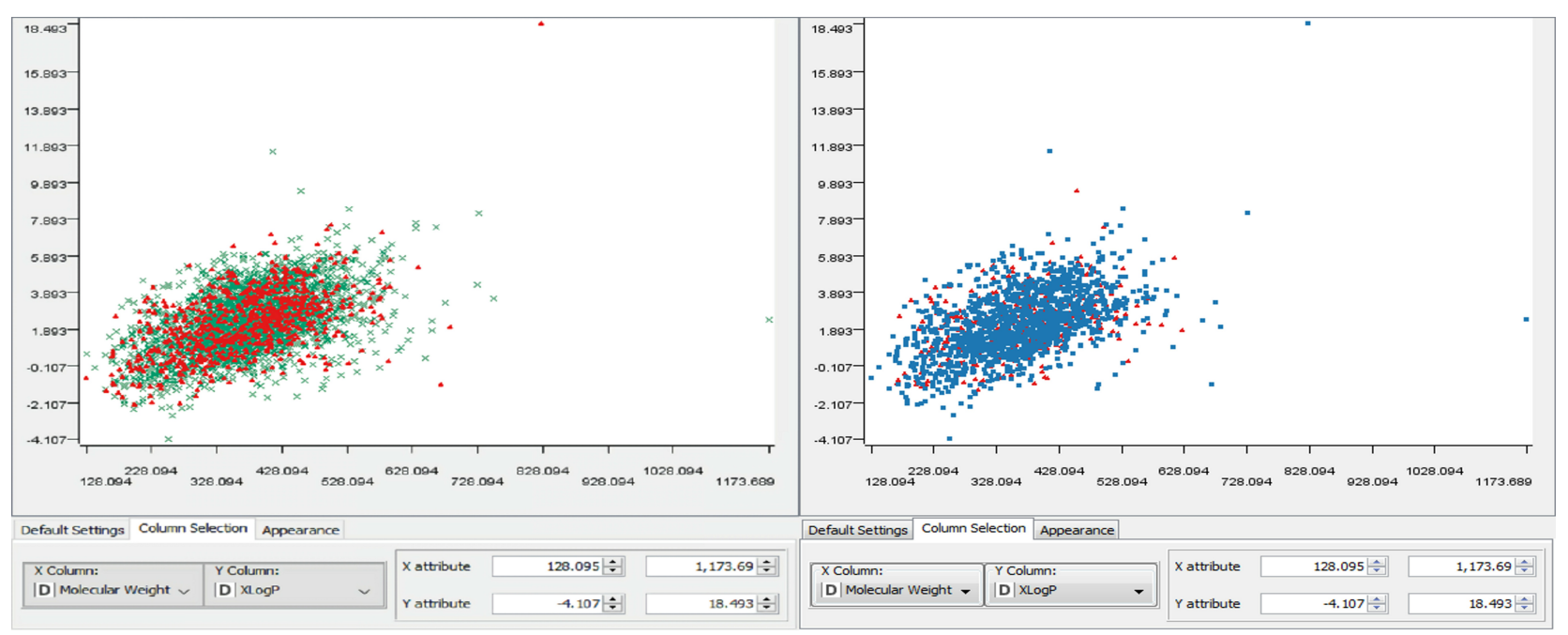

Figure 2

Chemical space training set versus test set (external validation) defined by MW and ALogP

(A) For the regression model (green $(x)$ is a training set, red $(\Delta)$ is a test set), and (B) for classification model (blue $(\square)$ is a training set, red $(\Delta)$ is a test set. 


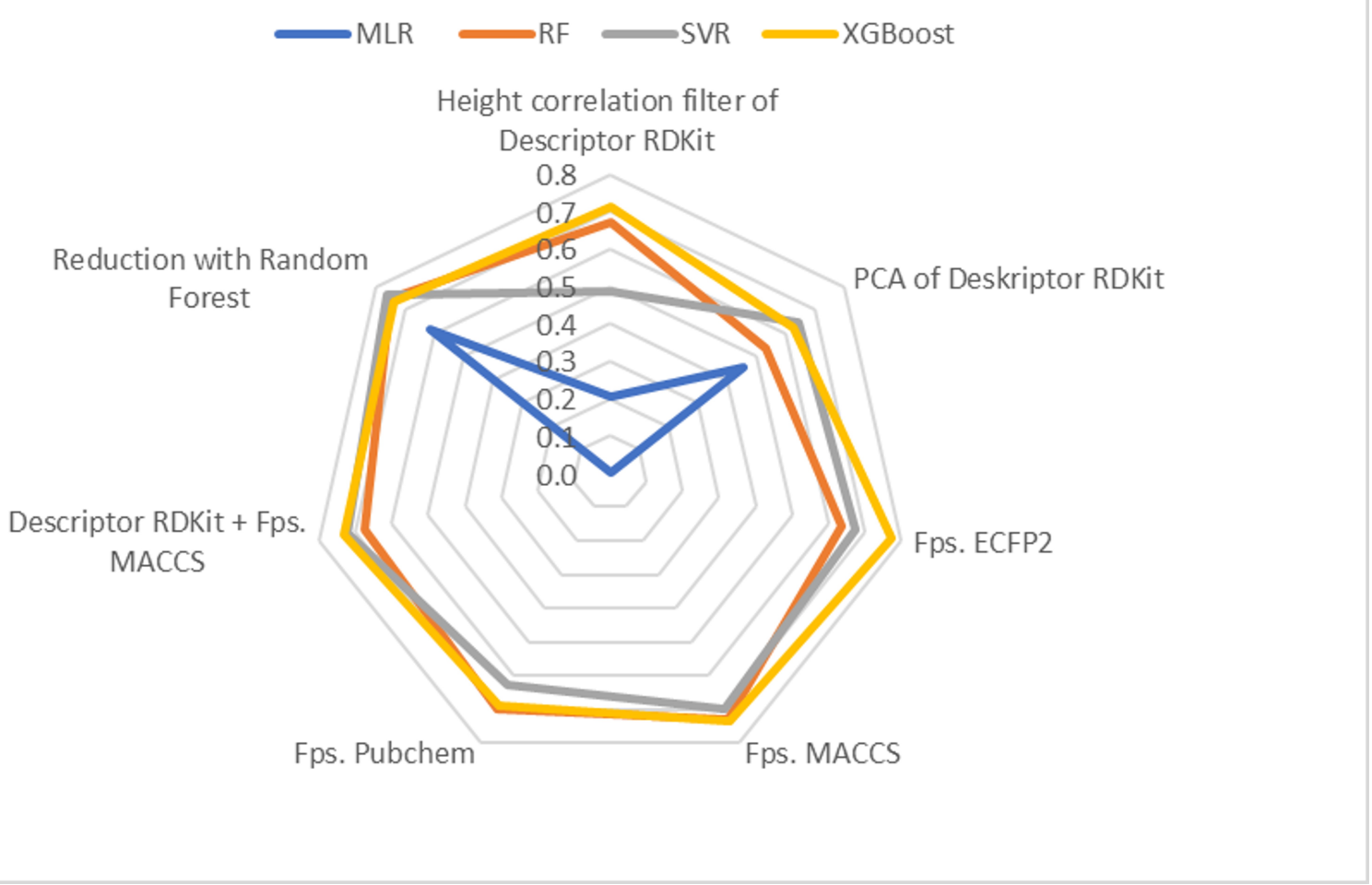

Figure 3

Feature selection. There are seven features developed to get the best method, using four learning models. 


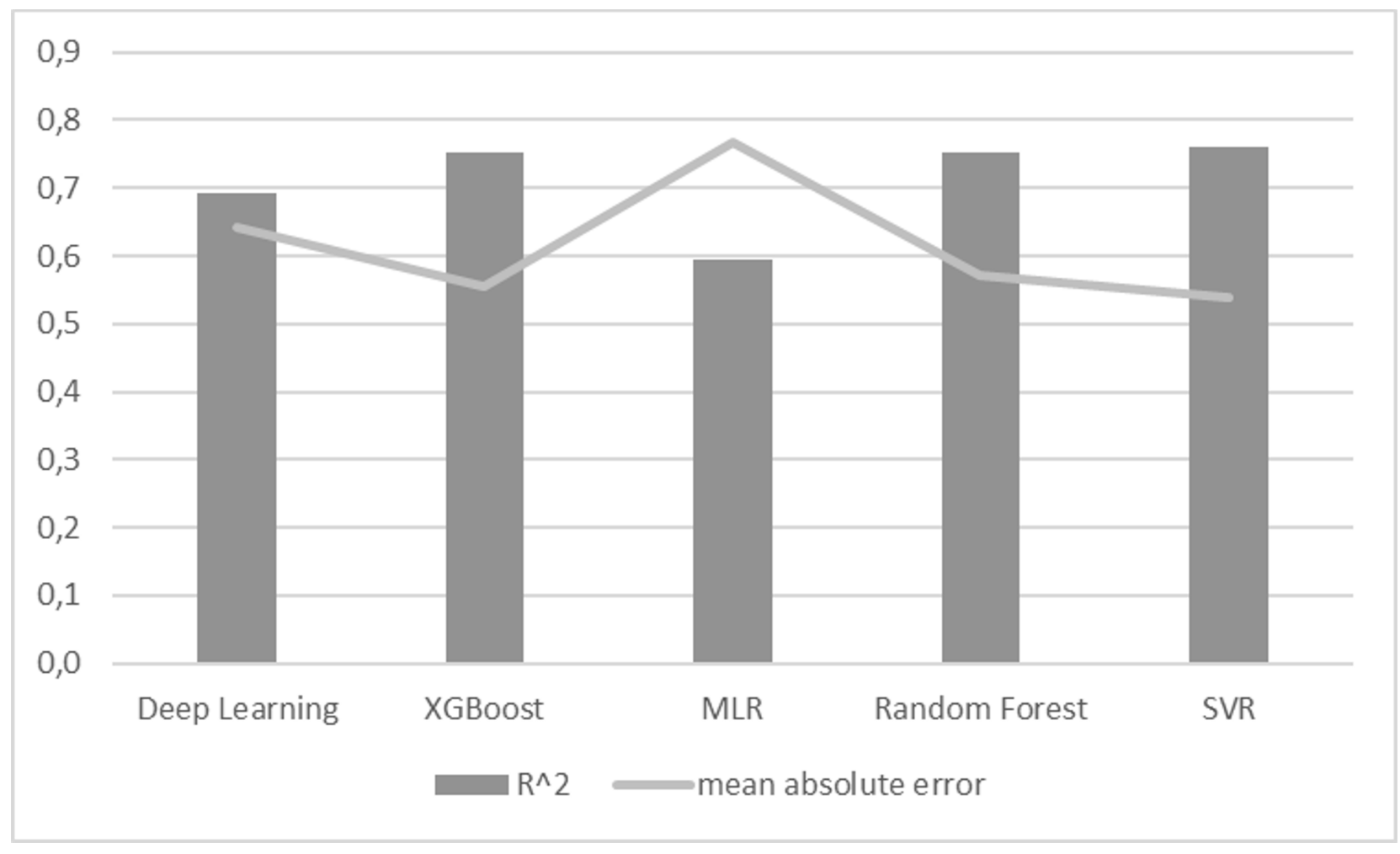

Figure 4

Internal validation results in the regression model The SVR model produces the best performance among other models with the lowest MSE 
a. From MolPort database:

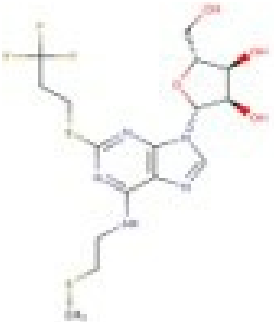

MP0001

prediction pIC $_{50} 8,4884$

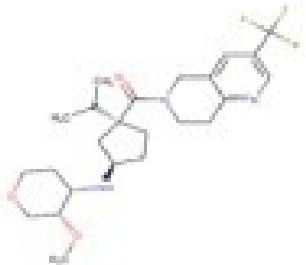

MP0002

prediction IC $_{50} 8,378$

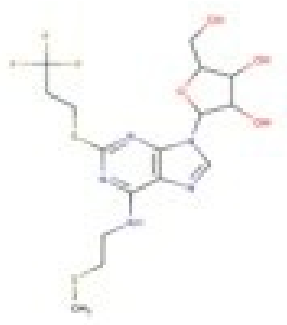

MP0002

prediction pIC $_{50} 8,326$

b. From ChEMBL database:

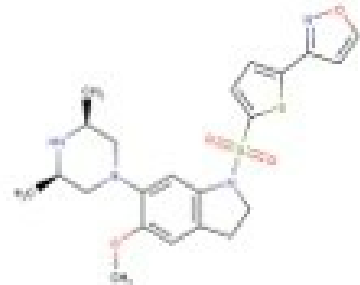

$\mathrm{CH} 0001$

prediction pIC $_{50} 9,1616$

c. From PubChem database:

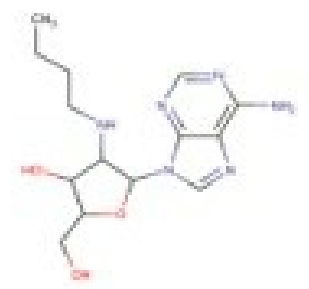

$\mathrm{PC} 0001$

prediction pIC $_{50} 7,9421$

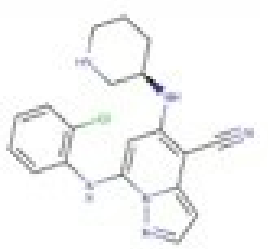

$\mathrm{CH} 0002$

prediction pIC $_{50} 9,105$

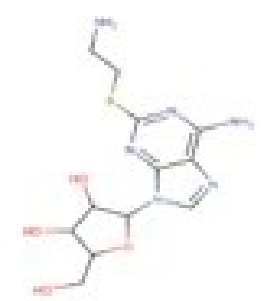

$\mathrm{PC} 0002$

prediction $\mathrm{pIC}_{50} 7,8961$

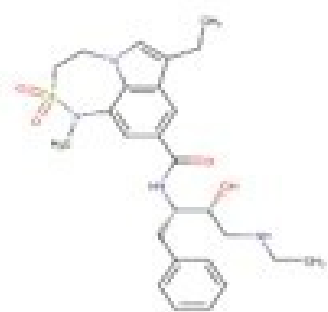

$\mathrm{CH} 0003$

prediction pIC $_{50} 9,061$

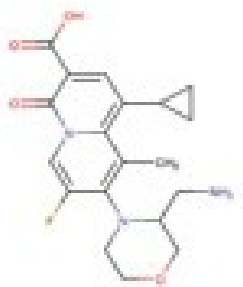

$\mathrm{PC} 0003$

prediction pIC $_{50} 7,7856$

Figure 5

Figure 5. Molecular structure of several hit compounds that was resulting from virtual screening 


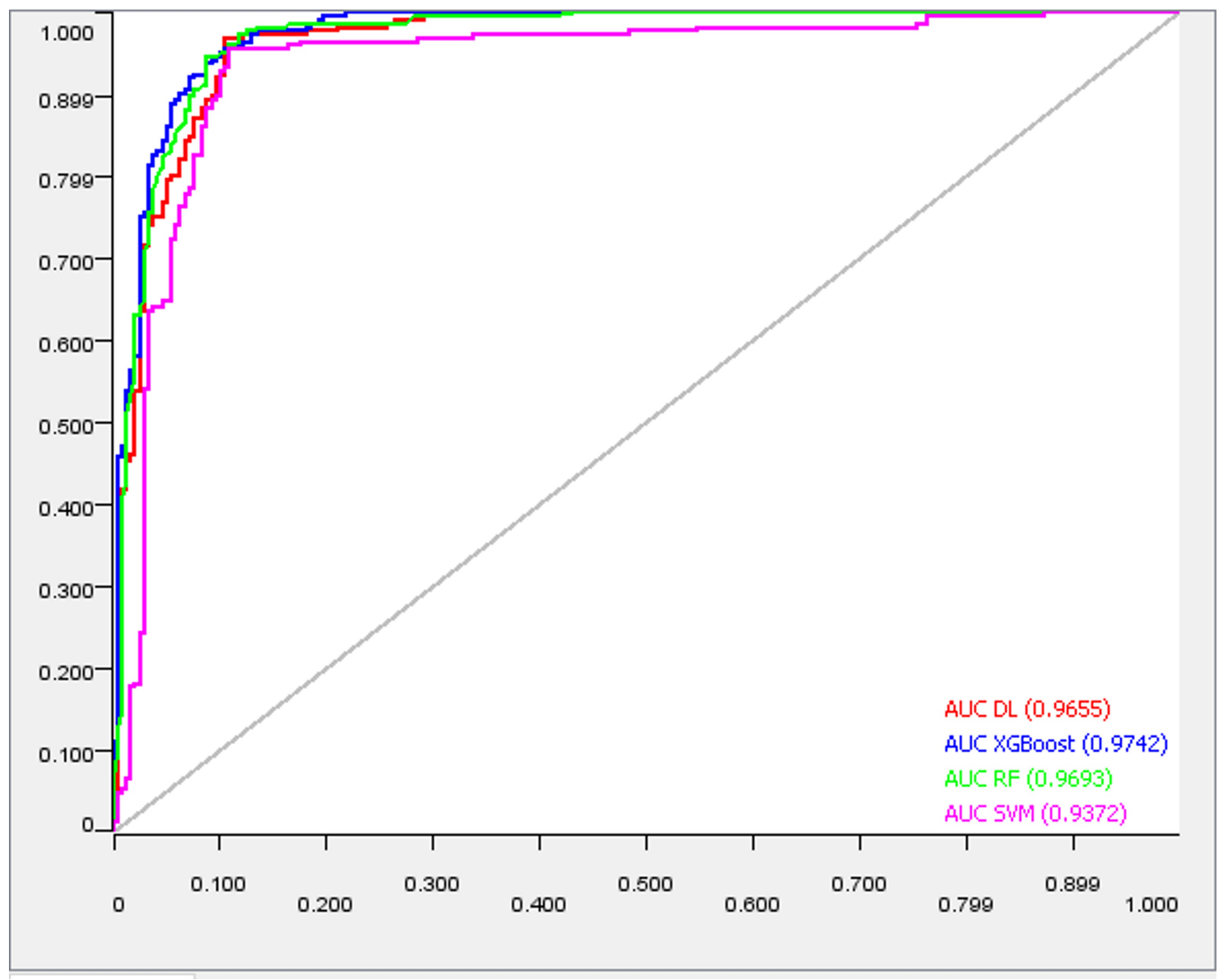

Figure 6

The ROC curve of the four classification models that developed 


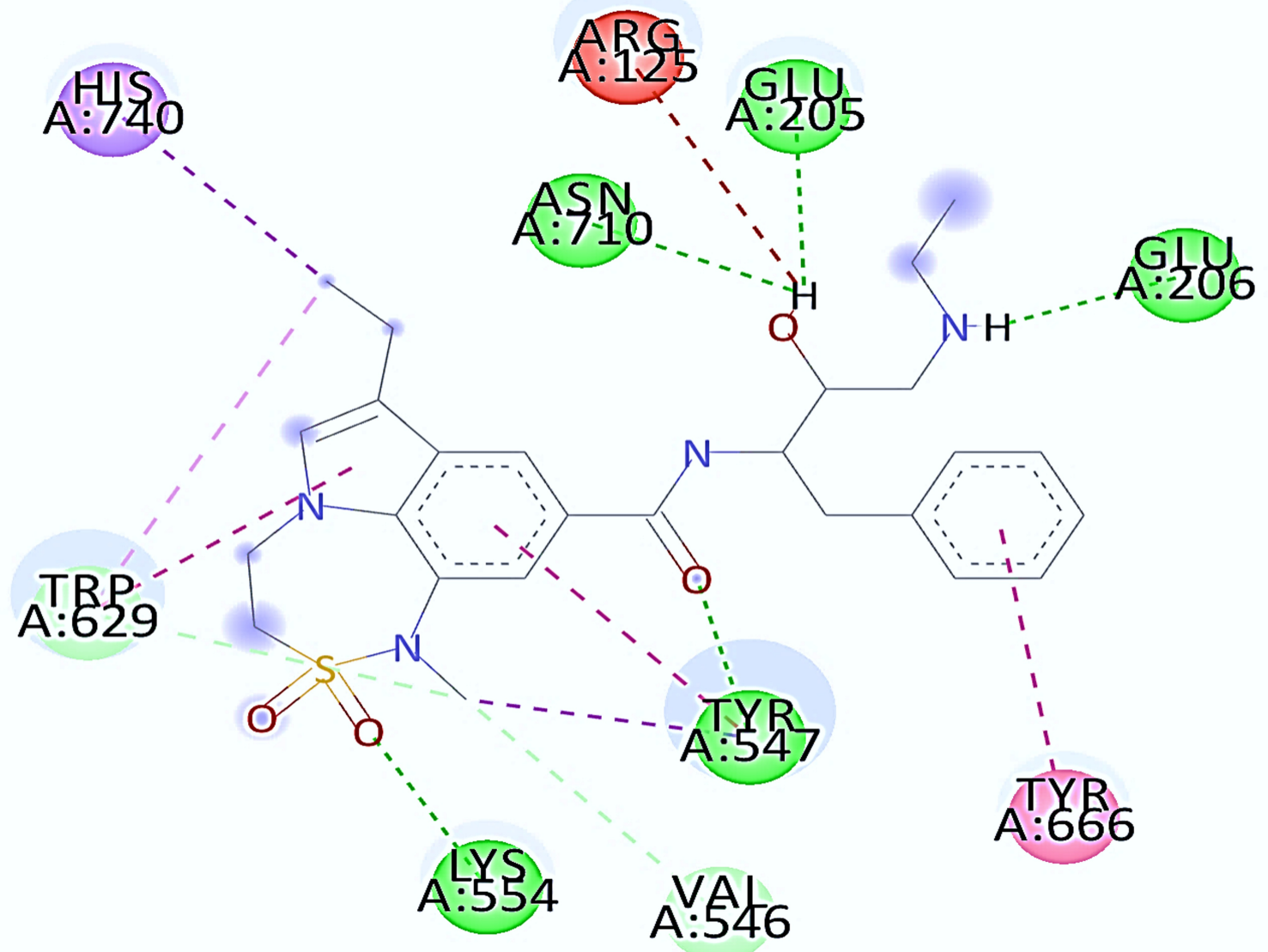

Figure 7

Unfavorable donor-donors interactions (in red) of hit compound CH0003 with DPP-4 (2ONC) crystal structure 


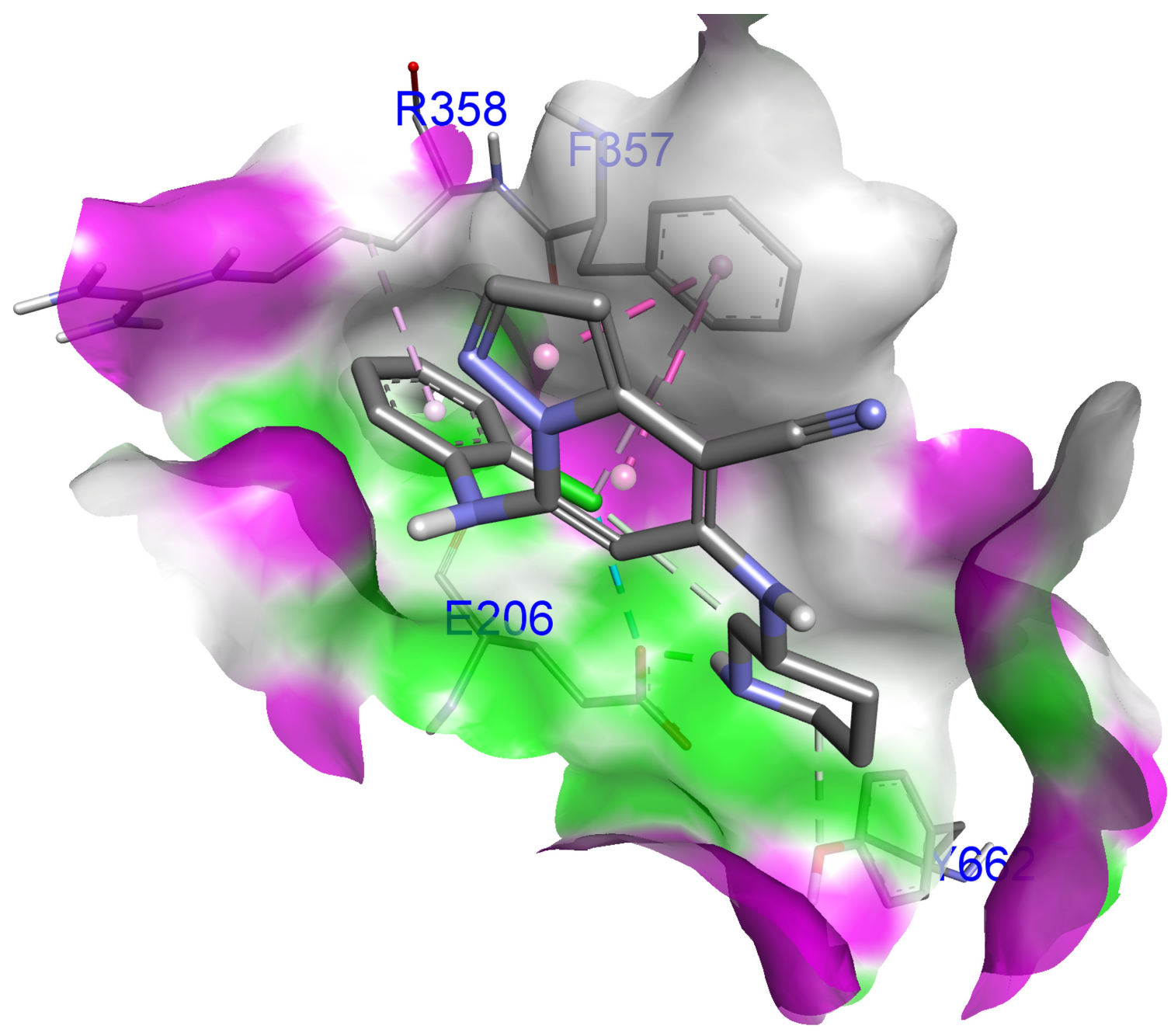

Figure 8

Visualization of the $\mathrm{CH0002} \mathrm{hit} \mathrm{molecule} \mathrm{on} \mathrm{DPP-4} \mathrm{(4PNZ).}$

\section{Supplementary Files}

This is a list of supplementary files associated with this preprint. Click to download.

Graphical Abstract.png

Suplementary File.rar 